\title{
Discovering America: Unpacking Popular Social Q\&A for Prospective Chinese International Students
}

\author{
Hon Jie Teo ${ }^{1}$ \\ ${ }^{1}$ New York City College of Technology, New York, United States \\ Correspondence: Hon Jie Teo, School of Professional Studies, New York City College of Technology, 300 Jay \\ Street, Brooklyn, NY, 11201, United States. Tel: 1-718-260-5373. E-mail: hteo@citytech.cuny.edu
}

Received: May 5, 2020

Accepted: September 1, $2020 \quad$ Online Published: December 26, 2020

doi:10.5539/ies.v14n1p1

URL: https://doi.org/10.5539/ies.v14n1p1

\begin{abstract}
The global pandemic and recent shift to online learning has heightened the need to better understand how to support international students, especially across online and virtual platforms. However, a review of literature reveals a paucity of studies dedicated to international student use of internet-based platforms and services for seeking information and sharing experiences. One avenue for further research is Social Question \& Answer Communities (SQACs), which are vast conduits of shared experiences and knowledge connecting hundreds of thousands of pre-application and pre-arrival students. In this study, content analysis was utilized to qualitatively observe the contents of the "Overseas Studies in The United States" section of Zhihu, and quantitatively count their features and characteristics. The study found that $58 \%$ of the questions and answers were devoted to Academic issues such as testing, admissions, learning and research, with another $13.0 \%$ on Crime, Law and Safety, and the remaining $29 \%$ of the questions were associated to a diverse array of topics associated with living and working in American society. The most popular answers were made up of mainly 4 main types: Sharing One's Experience $(32.0 \%)$, Advice $(26.0 \%)$, Opinion $(22.0 \%)$ and Critique $(15.0 \%)$. Content analysis of three main answer features, namely the use of Imagery, Digital Resources, and Social feature, indicated that the Advice and Critique answer types contain the richest variety of features and that question context, textual styles and use of digital resources are important factors for understanding the answer popularity in SQACs.
\end{abstract}

Keywords: social q\&a communities, international students, informal learning environments

\section{Introduction}

\subsection{Increasing Challenges of Studying in America}

In recent years, increased protectionism and barriers to immigration has impacted enrollment and raised widespread concern in the high education circles. The concern is not unfounded - international students bring significant financial and intellectual contributions to their host countries. Consisting of both undergraduate, graduates and visiting scholars, the number of international students has reached a total number of 1.17 million in March 2019 (ICE, 2019). According to the Institute of International Education (2019), international students contribute at least $\$ 42$ billion dollars, on an annual basis, to the American economy. According to the NAFSA: Association of International Educators (2019), the monetary expenses of international students indirectly supported over 458,209 jobs and contribute nearly $\$ 41$ billion of economic value to the US Economy. These economic contributions were made through expenditure of tuition fees and living expenses with the bulk of their expenses are primarily supported by funding through their personal funding and families, and represent a significant commitment towards higher education and their personal success.

According to UNESCO (2016) and ICE (2019), the People's Republic of China (PRC) sends the greatest number of post-secondary students overseas as compared to that of other countries. With respect to the United States, Chinese students from the PRC has reached an all-time high number with close to 370,000 undergraduate and graduate individuals enrolled in the American higher education institutions. This accounts for more than a third of all international students, which is more than that of any other country and is approximately half of the total number of the Chinese students pursuing overseas studies as international students in foreign countries (UNESCO, 2016). Raised in countries with distinct cultural and linguistic differences, the Chinese students bring with them diverse cultural and intellectual assets which position them to be potential intellectual assets for the United States. Their investment and effort do not go unwarranted. Upon completion of their education, the return of Chinese 
students to their home countries has allowed them to make significant contributions to the ongoing socioeconomic development of their home country (Cheung \& Xu, 2015).

\subsection{Challenges Faced by International Students}

In the American university and college classrooms, Chinese students face obstacles and ill-adaptation due to the differences in academic expectations and norms that contrast greatly with their pre-college educational experiences in PRC (Rawlings \& Sue, 2013). For instance, researchers compared the learning cultures in both countries, and found that American college experience emphasizes on collaborative group work whereas the Chinese pre-college experience mainly rely on testing and examinations (Chao et al., 2019; Heng, 2017). Their academic acclimation challenges are further exacerbated by the fact that international students come less prepared to meet the demands of freshmen writing classes and instruction in English (Lopez \& Bui, 2014), having most of their learning experiences conducted in Simplified Chinese language (Zhao \& Mawhinney, 2015). When burdened with additional English as Second Language (ESL) courses, they often face challenges in communicating verbally and in writing using a language other than their native language or mother tongue (Bertram et al., 2014).

In the American society, international students struggle with establishing and growing social ties with their American counterparts in addition to adapting to the local customs and norms (Liao \& Mei, 2014). Students who have arrived in their host country lack the necessary social support systems that native students have developed over time through their families and local communities. Further, the international students are expected to rely on a constrained social circle to prepare for their adjustment to new environment prior to arrival, which is not helped by an overtaxed group of staff and faculty members from the immediate college community (Heng, 2017). It is not surprising that it has been found that international students tend to experience anxiety and depression as a result of their academic challenges in their first few semesters of studies in the United States (Alpaslan \& Yalvac, 2017). This further supported the notion that academic success for international students is linked to the frequency of interactions with their immediate academic community and their sense of belonging to their universities (Glass \& Westmont, 2013). Given what Chinese students lack, online platforms such as virtual communities, social networking sites and social Q\&A communities become accessible avenues to expand their social ties.

\subsection{Perspectives of America}

The pursuit of an American education is a hefty investment that requires several years of academic preparation and financial resources ranging from tens to hundreds of thousands of dollars. However, Chinese parents are motivated in making the investment as they believe that the diverse America society and communities will foster the holistic development of the individual and the acquisition of a globalized world-view that are critical to personal and professional success (Chao et al., 2019). Chinese students believe that American academic and work programs such as internship, co-op and design projects provide them with well-deserved opportunities to gain workplace skills and industrial experience prior to graduation (Henze \& Zhu, 2012). Furthermore, Chinese students believe that America is one of the few places in the world where they have the freedom and support to hone their creativity and reasoning skills (Austin \& Shen, 2016). On the flip side, due to the widespread availability of firearms in the United States and the sheer size of the country, they are also concerned about personal safety, crime and (Nicholls, 2018). Chinese students are also apprehensive of the stereotypes of Chinese students held by their American peers, who view them as socially inept and irritating (Ruble and Zhang 2013). They are unsure if they will receive a reasonable amount of care and concern from professors and teachers in the United States due to differences in background and upbringing. More recently, frictions between China and America have led them to be unsure if criticism of their birth country will hinder their relationships with their peers (Hail, 2015).

\subsection{Student Use of Social Question and Answer Communities (SQACs)}

Social Question and Answer Communities (SQACs) are thriving social groups hosted on online platforms where many students coalesce to pose questions, collectively generate solutions, and share knowledge on an online web platform (Adamic et al., 2008; Chen \& Hew, 2018; Gazan, 2015). Communication is initiated by a questioner who poses a question that is openly accessible to all or seeks help by submitting a request to the community (Chen \& Hew, 2018). In response to posed questions in SQACs, answerers could propose an answer in form of a shared knowledge, experience, solution and referral to another person (Chen \& Hew, 2018). The widespread use of SQACs can be traced to its persistent online presence for immediate access, preservation of user and visitor anonymity, diversity of content to support a range of information needs, the openness of the system in capturing and storing all responses. It has emerged as a major knowledge source for its capacity for collective intelligence, made possible by its facilitation of posting questions, collecting answers and evaluation of answers (Chen \& How, 2018). Yahoo Answers, as one of the most visited SQAC in North America, has over 100 million contributors and 25 million questions. Specifically, in Yahoo Answers, members with higher levels of expertise actively share their 
knowledge by helping each other to solve problems, telling stories of personal experienced events, and debating issues based on evidence and consensus (Adamic et al., 2008).

College students frequently seek information on SQACs related to navigating the college experience as well as everyday living matters due to the perceived ease, increased familiarity, and built-up social ties over time (Fang et al., 2019; Kim et al., 2014). International students have diverse range of information needs (Chung \& Yoon, 2017; Hail, 2015) and that they typically seek desired information from a wider variety of web-based resources such as online forums and SQACs in addition to web articles linked from web search engines. The SQAC stands out due to its more interactivity between the initiator and the responders, together with the potential gains due to repeated socializations (Guan et al., 2018). The advantages of using SQACs are multifold for learners from differing needs, interests and backgrounds. The positive effects include a learner's improved sense of belonging, perceived usefulness to others and heightened intention to help newcomers develop stronger social ties (Fang et al., 2019; Guan et al., 2018). Other advantages of participating in SQACs are its perpetually-maintained online presence that grants unlimited access to capturing and storing all responses, one's ability to remain anonymous, a wide variety diversity of content targeted at addressing common issues and unique problems previously faced by others (Chen \& How, 2018). Other appeal of SQACs is in its low-cost accessibility, whereby a rich archive of articulate interaction that other readers can read and draw experience regardless of their location and time of access (Gazan, 2011). The instantaneously searchable and accessible archives of crowdsourced "wisdom" reduce the need for duplicate questions and allow learners to channel their efforts to problem solving and troubleshooting tasks (Mamykina et al., 2011). Another appeal of SQACs is its allowance to resolve intricate and complex questions under anonymity without any exposure of personal information as contrasted against social media and social networking platforms (Yang et al., 2011). This attracts a wider audience and a bigger pool of willing participants who value their privacy.

\subsection{Purpose of Research}

The overarching focus of the research is to deepen the understanding of the SQAC use by Chinese students in the earlier stages of pursuing overseas education in the United States. This is attained by evaluating the characteristics of contributions and activities, and by identification of the most upvoted Q\&A based on their discourse, interactions and artifacts. The goal of the study includes an investigation of the types of questions asked, the most upvoted answers and the features of such answers. The emphasis is on a large online group titled "Overseas Studies in United States" of the largest Chinese SQAC, Zhihu, and guided by the research question: and the characteristics of answers most well-received by prospective Chinese international students on the SQAC?

The study was guided by the following research questions:

1) What types of questions and answers were prevalent in the most popular Q\&A posts?

2) What are the characteristics of answers most well-received by prospective Chinese international students?

\section{Method}

Zhihu, one of the most popular SQACs in China accessible through http://www.zhihu.com, was the site of study. In each topic area, only 1000 most upvoted questions were accessible to the public. At the commencement of the study, there were a total of 133,972 subscribed members who have asked a total of 62,381 questions in this section of Zhihu. As with most SQAC platforms, a typical Zhihu SQAC Web page, the page includes information the date when the question was asked, the date of the answers and the alias of the involved contributors. The page also tracks and displays the number of upvotes received and the usernames of the participants. The questions and the accompanying most upvotes responses were downloaded onto a spreadsheet to be numbered, organized and classified. The selection of the most popular answers is primarily based on the visible count of "upvotes" by members of the community who have viewed or participated in the discussion. Next, the questions and responses are numbered and analyzed based on the principles of grounded theory, based on an emergent and iterative process as described earlier. The textual contents of the questions and answers were analyzed in Chinese whereas the results of the analytical process are described and documented in English. On the other hand, the quantitative steps are carried out using descriptive statistics to calculate the frequency and intensity of activities in relation to the discovered themes. The questions and answers were written in the period spanning 1st January 2015 to 30th October 2018.

\subsection{Data Collection}

Systematic capture of all visibly accessible top upvoted questions and answers was carried out based on a Python script which automates the download of the questions and answers in September 2018. This resulted in the 
download of 1,000 web pages in HTML format. Additional manual checks were made to ensure that the webpages were intact and contains a complete list of written contents, web links to external resources, and embedded digital resources. The final step of data processing was initiated to extract the contents each message on the web pages and transfer other quantifiable information such as the number of upvotes, the number of characters, message content and the time of posting each message into a database.

\subsection{Research Design}

Content analysis, an empirical method based on an exploratory process with an inferential purpose (Krippendorff, 2004), informs the research approach for this study. Content analysis is typically used to analyze the details of online messages and uncover recurring themes in studies of online discourse based on qualitative and quantitative components (Lai \& To, 2016). The qualitative component is focused on making sense of, identifying and understanding the contents of Q\&A, in order to categorize the posts into themes (Charmaz, 2006) while, on the other hand, the quantitative aspect focuses on counting the intensity or frequency of key word phrases, concepts and recurring themes (Krippendorff, 2004; Lai \& To, 2016). In this approach, the selected content is to be numbered, analyzed and categorized using ground theory procedures of open, axial and selective coding (Corbin \& Strauss, 2007). Abiding by a confidence interval of $50 \%$ and margin error or $5 \%, 214$ out of the 1000 upvoted Q\&As were selected to be examined from the sub-section of "Overseas Studies in America" of Zhihu.com. Upon examination, 14 of the topics were of narrative nature rather than of a questioning nature, and were omitted from data analysis. The analytical process of open coding is carried out by the two coders reading each question and the answer in Chinese, with a focus on highlighting the most salient phrases, words and concepts, resulting in the 37 open codes based on examination of practices, the ongoing processes and the developing trends. Inter-Coder Reliability is measured by the Krippendorff's Alpha and reached 0.823 , suggesting an acceptable level of agreement between the coders. Then, the open codes are consolidated and categorized into axial code groups before they are refined through the selective coding process, yielding a total of 8 themes for question topics and 6 answer types. Upon the completion of qualitative coding, quantitative content analysis was used to statistically tally the frequencies of occurrences of 15 parameters related to the use of artifact, words and features in the dimensions of Images, Digital Resources, and Social Features.

\section{Results}

\subsection{Question Topics}

As summarized in Table 1, the analysis of the topics and written contents of the questions indicate that (58.0\%) of the questions were contextually Academic: Learning at the University (26.0\%), Admissions and Testing (25.0\%) and Faculty Research (7.0\%). Questions related to Academic and Learning (26.0\%) appeared most frequently, followed by Admissions and Testing (25.0\%). An example of a question for Admission and Testing is "How do I improve my writing for TOEFL? I want to improve my writing standard, especially for the section of Integrated Writing." The comparison of Learning at the University (26.0\%), Admissions and Testing (25.0\%) and Faculty and Research (7.0\%) suggests the question topics were viewed mostly by pre-application and pre-arrival students, rather than graduate students. Additionally, this suggest that most of the questions originated from China, as they are related to the pre-application and pre-arrival academic matters related to application, admissions and enrollment in higher education institutions in the United States. Questions about Faculty and Research (7.0\%) were typically oriented towards the discussion of faculty members, their research and whether they were good mentors for graduate students.

Table 1. Frequency counts and examples of question topic

\begin{tabular}{ccc}
\hline Question Topic & Frequency $(\mathrm{n})$ & Percentage (\%) \\
\hline Admissions and Testing & 52 & 25.0 \\
Learning at the University & 50 & 26.0 \\
Research and Professors & 14 & 7.0 \\
Crime, Law and Safety & 26 & 13.0 \\
Career and Jobs & 14 & 7.0 \\
Overseas Lifestyle & 22 & 8.5 \\
Society and Culture & 17 & 11.0 \\
Immigration & 5 & 2.5 \\
\hline
\end{tabular}

The next most frequently occurring questions were not of an academic nature but rather were related to Crime, 
Law and Safety (13.0\%). These discussions centered on issues, concerns and questions with the campus environment, guns, personal safety and crime prevention. Its relatively high frequency is not surprising given the differences in perceptions towards the use of firearms, rule of law and general safety conditions in both countries. The remaining $29.0 \%$ of the questions were associated to a diverse array of topics associated with living and working in American society: Society and Culture (11.0\%), Overseas Lifestyle (8.5\%), Career and Jobs (7.0\%) and Immigration (2.5\%). Overseas Lifestyle (8.5\%) was related to general matters to living standard and comfort in the United States, whereas questions for Society and Culture (11.0\%) were related to societal practices and customs of the United States. Career and Jobs (7.0\%) questions were asked about job opportunities and prospects across an entire spectrum of degree specializations, and Immigration (2.5\%) were focused on immigration matters such as visa applications, Green Card application, Occupational Curricular Training (OPT) and American citizenship.

\subsection{Answer Types}

As summarized in Table 2, analysis of the responses and the contents indicate four types of responses made up almost all (95.0\%) of the examined answers. The most frequently occurring answer type is one that involves the Sharing One's Experience (32.0\%) where the answerer provided a narrative description or opinion that drew from the personal lived experience of the answerer. The second most common type of response was the providing Advice (with a proposed course of action) to the questioner (26.0\%). The third most frequent answer type is Opinions $(22.0 \%)$, where answerers will offer interpretation of an issue or information related to the question asked, whereas the following fourth most frequently occurring answers involves a written Critique (15.0\%), whereby the answerer will write an analytical assessment of a topic related to the question asked. The remaining $5.0 \%$ of the answer types consist of Sharing Others' Experiences (3.0\%) and One-Liners (2.0\%).

Table 2. Frequency counts and examples of answer types

\begin{tabular}{ccc}
\hline Answer Types & Frequency (n) & Percentage (\%) \\
\hline Advice (With Course of Action) & 52 & 26.0 \\
Shares One's Experience & 65 & 32.0 \\
Shares Others' Experience & 6 & 3.0 \\
One Liners & 3 & 2.0 \\
Critique & 29 & 15.0 \\
Opinions & 45 & 22.0 \\
\hline
\end{tabular}

\subsection{Features and Characteristics of Answers}

Table 3 provides a summary of the mean number of typed characters featured in six types of answers: 3,256 for Advice, 2,406 for Critique, 2,065 for Sharing One's Experience, 1,740 for Sharing Others' Experiences, 1,462 for Opinion and 34 for One Liners. One observation that stands out is that Critique had more than a thousand more characters on average as than opinions whereas Sharing One's Experience had approximately 300 words than Sharing Others' Experiences - indicating that critique were in-depth writings whereas experience falls short in terms of word count and textual depth. In addition, the mean number of upvotes is as follows: 4,792 for Advice, 4,561 for Sharing One's Experience, 4,372 for Sharing Others' Experiences, 3,703 for Opinion, 2,406 for Critique and 1,781 for One Liners.

Table 3. Frequency counts and examples of question topic

\begin{tabular}{ccc}
\hline Answer Type & Mean Number of Characters & Mean Number of Upvotes \\
\hline Advice & 3256 & 4792 \\
Critique & 2406 & 2932 \\
One Liner & 34 & 1781 \\
Opinion & 1462 & 3703 \\
Sharing One's Experience & 1740 & 4561 \\
Sharing Others' Experiences & 2065 & 4372 \\
\hline
\end{tabular}

The distribution of the types of images used in the answers and its frequency of appearance per answer is shown in Figure 1. Overall, most of the images were used less than 0.2 times per answer for the categories of Memes, Media, 
People, Objects and Places. It is observed that screenshots, originating from computers and smartphones, were the most used images whereas Book Covers and Graphs were the least used for most answer types. There are variances in the types of images used. For instance, graphs were heavily used as support materials in Critiques, whereas book covers and screenshots were more frequently used in Advice to direct usage of certain applications and books compared to other answer types. These suggest that Advice commonly involved a recommended course of action prescribed directions and actions to use specific cell phone and computer applications, and references to the use of specific publications and books. Screenshots were also commonly used for Sharing One's Experience (0.24), Sharing Others' Experience (0.17), Critique (0.31), Opinion (0.2) and Advice (0.48), which highlight the utility of screenshots over embedded resources and webpage linkages as a way to more richly describe complex issues or contexts of an answer.

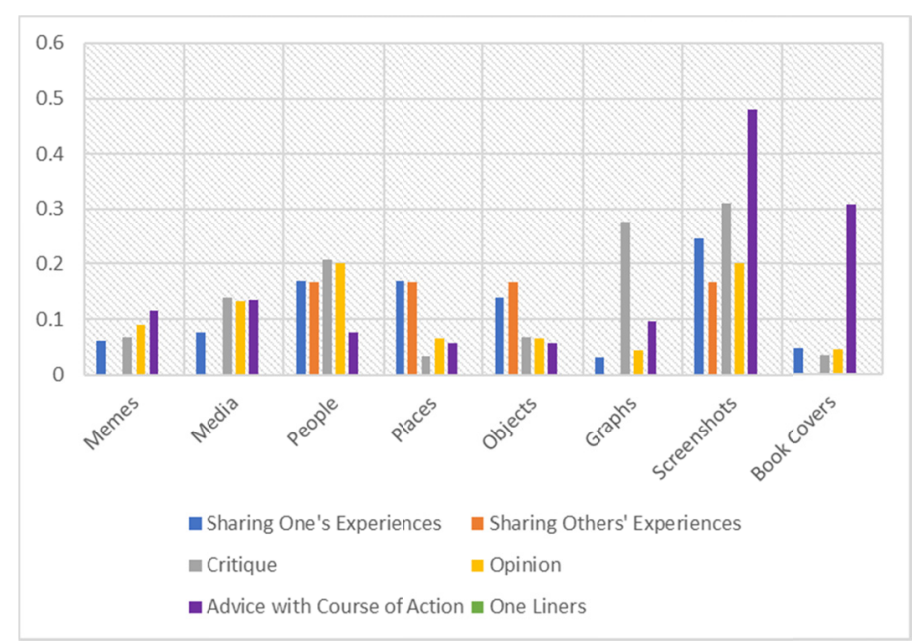

Figure 1. Frequency of use of imagery use per answer

Figure 2 shows the distribution of the digital resources provided by answerers and the frequency of appearance per answer. The distribution varies widely based on the type of answers. It is found that embedded videos and documents were rarely featured in the answer types excluding Advice (embedded videos at 0.06 and documents at 0.1 ), which is suggestive of the presence of highly diverse resources prescribed as part of the recommended course of action and directed plans in Advice. Another observation that stands out is that links to internal Zhihu webpages were not used at all in Sharing Others' Experiences although they were used in other answer types such as Sharing One's Experience (0.24), Sharing Others' Experience (0.17), Critique (0.31), Opinion (0.2) and Advice (0.48). Citation of Works is one feature that shows consistency in its frequency of occurrence; with a range of $(0.09$ to 0.17 ) in the all the answer types other than One Liners. Another finding that stands out is that External Webpage Links were frequently featured in Sharing Others' Experiences but on the other hand Internal Webpage Links were not at all used in Sharing Others' Experiences. This observation suggests that others' experiences discussed in one's answers were not derived from other Zhihu answers, but rather they were based on the personal experiences and accounts of the answerers' academic, professional and social experiences. 


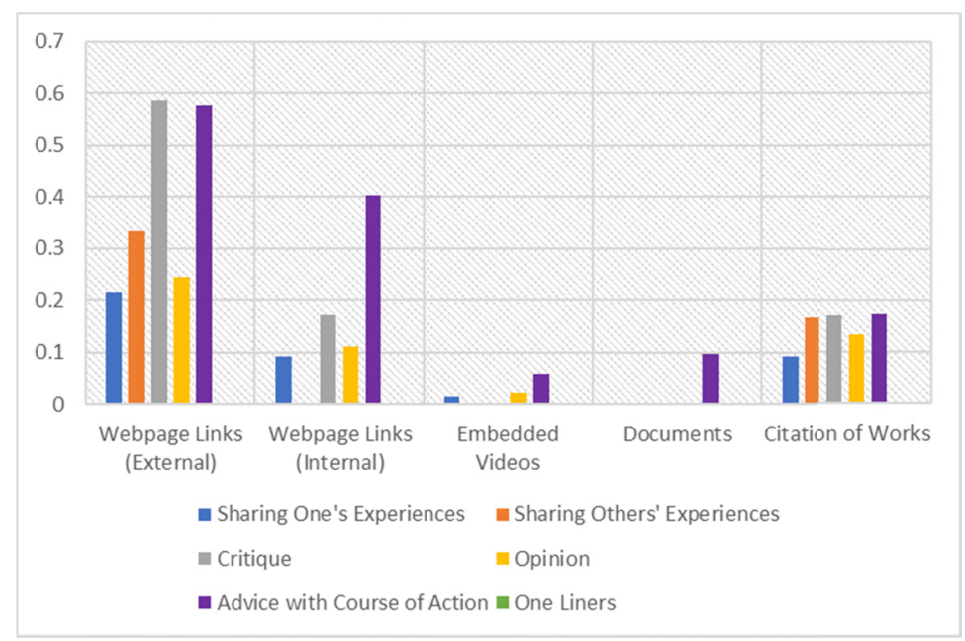

Figure 2. Frequency of use of digital resources per answer

The distribution of Social Features in the various answer types and its frequency of appearance per answer is illustrated in Figure 3 and it can be seen that social features vary in its use $(0.08$ to 0.33$)$ across all answer types excluding One Liners. One observation that stands out is that Sharing Others' Experience had the most References to Other Members (0.17) and Contact Information (0.33) which included inclusion of personally identifiable information such as email addresses and social media accounts. This in turn support the notion that answers containing Sharing Others' Experience afforded more opportunities for forging social connections and building relationships in the community.

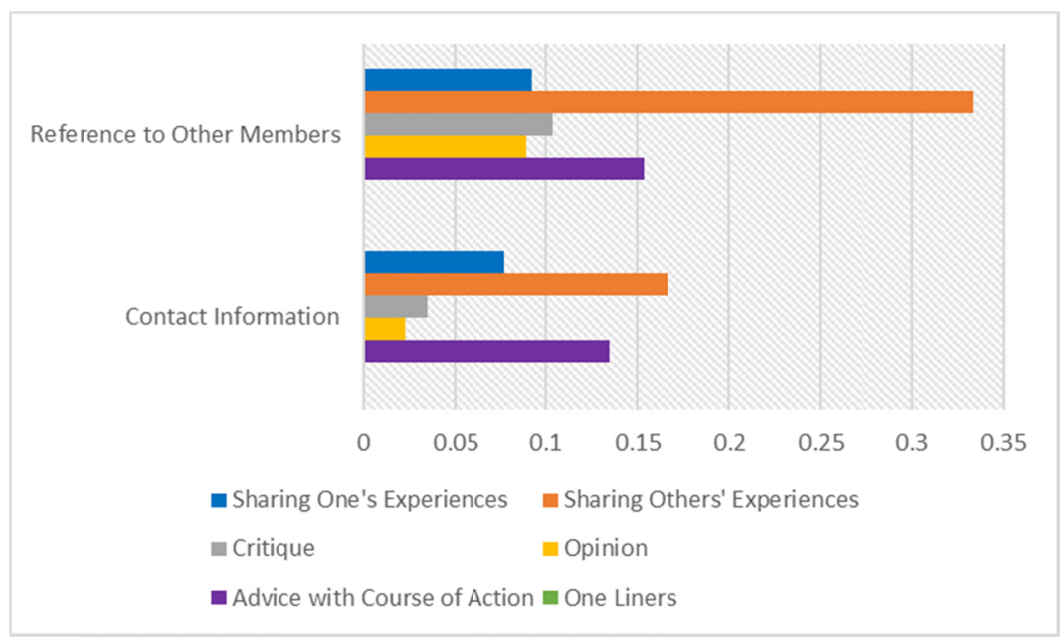

Figure 3. Frequency of use of social features per answer

\section{Discussion}

The findings indicated that Q\&A in the SQAC were not limited to academic or education discussions as the title of the community suggests - "Overseas Education in the United States". Topical analysis of the Questions found that $58.0 \%$ of the questions were of an Academic nature, and $42.0 \%$ were not, suggesting that the community brought together participants with a range of interests and expertise level to engage in information sharing in a broad range of topics and domains. This finding is consistent with documented challenges of pursuing overseas education, where international students typically face a broad range of problems and barriers culturally, linguistically, and academically (Bertram et al, 2014; Yan \& Berliner, 2011). For the non-academic questions, topics related to Crime, Law and Safety (13.0\%) appeared most frequently and centered on issues, concerns and questions with the campus environment, guns, personal safety and crime prevention. This result agrees with recent studies that established 
safety concerns as a major consideration for selecting overseas education destinations (Nicholls, 2018), and its relatively high frequency is not surprising given the differences in perceptions towards the use of firearms, rule of law and general safety conditions in both countries (Cretacci et al., 2018). The Chinese students were also interested in questions related to the Overseas Lifestyle (8.5\%) as well as Society and Culture $(11.0 \%)$ in the United States as these questions will allow them to discover more about their future way of lives upon arrival at their host country (Li et al., 2017; Xu \& Mocarski, 2014). When it comes to an important end goal of their education, jobs, the relatively low number of discussions related to Careers and Jobs (7.0\%) can be traced to an increase in returning Chinese students who target career corporate opportunities in China rather than the United States (Cheung \& Xu, 2015). The rest of the questions were of an Academic nature (58.0\%), and the results are similar to previous studies showing the popular use of SQAC amongst undergraduates to seek background information, find solutions to problems and obtain others' experience to navigate the college experience and achieve academic success (Kim et al., 2014; Sin et al., 2015). Overall, the findings align with the documented acculturation challenges and difficulties for international students (Bertram et al., 2014; Yan \& Berliner, 2011). While the Zhihu community appears to be slated for international students seeking information regarding overseas education in the United States without any explicit reference to overseas living or working, the topics appear to cover multiple aspects of preparing to live, study and work overseas in the United States. The findings give support to recent studies that reported SQACs as conducive environments that facilitates a diverse breadth of Q\&A focuses in the realm of the stated knowledge domain, and that its diverse Q\&A range provides an crowdsourced pool of resources that is accessible to all community members if they were to make the effort to search and read the active and archived Q\&A contents (Chen \& Hew, 2018; Fang \& Zhang, 2019; Liu \& Wang, 2016). Therefore, the results represent a significant opportunity to understand numerous issues and challenges face by pre-application and pre-arrival Chinese students to the United States: process of admissions for overseas education, exceling in academic coursework, graduate research, career considerations and living in the United States. This is made possible as the results enabled insights into answering type that are well-received and popular, or the answer features that garnered the most attention from other members of the SQAC.

Content analysis of the answer types yielded four types of responses that made up almost all of the examined answers (95\%), which add to the existing literature on social participation and technology-mediated communication in SQACs. The most frequently occurring answer type is Sharing One's Experience (32.0\%) where the answerer shares his or her personal lived experience by providing a narrative description or opinion that drew from a lived personal experience of oneself by providing a narrative account of an incident or situation. The finding lends support to recent studies highlighting the prevalence and commonality of experience sharing in SQAC (Abelmann \& Kang, 2014). The second most common type of response was the providing Advice (26.0\%) with a detailed course of action and it was often observed in the data analysis process that several answerers appear to display extensive domain knowledge to craft a proposed list of actions despite not stating how they came to acquire this knowledge. Such behavior can be explained by the formation of answering practices and discussion cultures over time in SQAC (Fang \& Zhang, 2019; Pata et al., 2016). The third most frequent answer type are opinions $(22.0 \%)$, where answerers will offer interpretation of an issue or information related to the question asked, whereas the following fourth most frequently occurring answer type involves a written Critique (15.0\%) containing an analytical assessment of a topic related to the question asked. The pervasiveness of these answer types is in line with studies that suggest that questioners typically seek answers that are complete and analytically reviewed as they suggest a degree of verification, accuracy, and vetting by other members of the SQAC (Finchman, 2011; Fu et al., 2019). These findings highlight the varying degree of answer receptiveness in the community and unsurprisingly, providing askers with advice with a suggested path of action and sharing ones' experiences are highly popular compared to one liners and critiques which may not necessarily possess information to fully address the issues and problems posed by questioners.

The analysis of 15 features spanned three categories: Imagery, Digital Resources, and Social Features. Findings indicated that answer type Advice, among the four main answer types, had the highest mean number of features across the categories, expectedly due to the nature of on providing a course of action which typically includes a variety of artifacts, textual and linguistic qualities to structure and emphasize one's answer. This result refutes the more widely-accepted observation that social intentions and recognition power most answers in SQACs (Liu \& Jansen, 2013; Pata et al., 2016). Critiques, derived from an analytical perspective, were the next most upvoted answer type and the feature counts were mostly slightly lower than Advice. However, Critiques had higher uses of features that requires a higher degree of numerical and contextual information, such as graphs and webpage links. This result echoes findings in similar studies where explanative answers were found to require additional feedback and improvements with regards to the use of resources (Erens et al. 2018, Teo et al., 2017). One Liners have on average least features due to its length and content depth although its presence in online communities add to the 
diversity of perspectives and affords opportunities for fostering camaraderie (Ren et al., 2007). Another answer type that stood out is Sharing Others' Experiences, whereby sharing of a third-party account of others' experiences led to the limited use of digital resources and linguistic features and conversely, extensive sharing of social references and contact information. This result is significant in the sense that several studies have focused on large datasets with statistical and datamining techniques (Adamic et al., 2008; Mamykina et al., 2011; Vasilescu et al., 2014), which restricted their ability to discern between sharing one's personal experience and sharing others' experiences. It was further understood that while online questioning and answering in this setting contain a variety of characteristics, the richness of the features could vary across the style of answering. The studied SQAC has gained much by being receptive and open in support of integration of multiple perspectives and self-directed sharing. This is evident in the study where the community perceived the most useful posts as those that shared personal experience and a planned course of action, and while it requires additional efforts in developing and evaluating one's answer, it could only strive in an open community receptive of diverse inputs and skeptical of conformity. The results also highlight a unique issue with answering in that those who know more and are more experienced are more able in selecting the best approach to develop sought-after answers, as these answerers can draw from their prior lived experiences and repertoire of knowledge including their actual experience of studying in American Universities and immersing in the culture of United States (Chung \& Yoon, 2017; Heng, 2017).

This study has several implications for research and practice, as it presents an extension of related studies concerning the use of online communities by Chinese international students prior and during their application to pursue higher education in the United States. The results offer crowd-sourced insights to administrators of college-preparatory schools or high schools in China seeking to improve or maintain their current approach of disseminating of best practices of application to American universities. Higher education institutions in the United States can benefit from the incorporation of crowd-sourced data from social networking sites and SQAC in addition to their internal evaluation instruments to cultivate educational programs or student services that can address the most challenging questions faced by international students upon their arrival at their host universities. The understanding about well-received answer types and question topics acquired as part of this study can guide recruitment and admission staff and practitioners in developing appropriate informational resources to better support international applicants to undergraduate and graduate programs in the American Universities. On the other hand, several opportunities exist for researchers to understand the effects of social participation on the actions and dispositions of the international students, through either longitudinal or intervention studies. Significant opportunities are available for researchers who are well-versed in the methods of statistics and data-mining and interested in developing learning analytic models to study discourse and interactions on SQACs at a large scale. For researchers seeking to advance the understanding of international student use of informal online communities, additional studies are needed to develop specialized instrumentation and learning analytics that can deepen our understanding of the context and significance of shared information, experience and knowledge on SQACs for international students.

\section{References}

Abelmann, N., \& Kang, J. (2014). A fraught exchange? US media on Chinese international undergraduates and the American university. Journal of Studies in International Education, 18(4), 382-397. https://doi.org/10.1177/1028315313479852

Adamic, L. A., Zhang, J., Bakshy, E., \& Ackerman, M. S. (2008, April). Knowledge sharing and yahoo answers: everyone knows something. In Proceedings of the 17th international conference on World Wide Web (pp. 665-674). https://doi.org/10.1145/1367497.1367587

Alpaslan, M. M., \& Yalvac, B. (2017). Exploring the anxiety state of the science majoring international graduate students with conditional acceptance in the United States. International Journal of Research in Education and Science, 3(2), 614-623. https://doi.org/10.21890/ijres.328092

Austin, L., \& Shen, L. (2016). Factors influencing Chinese students' decisions to study in the United States. Journal of International Students, 6(3), 722-732.

Bertram, D. M., Poulakis, M., Elsasser, B. S., \& Kumar, E. (2014). Social support and acculturation in Chinese international students. Journal of Multicultural Counseling and Development, 42(2), 107-124. https://doi.org/10.1002/j.2161-1912.2014.00048.x

Bodycott, P., \& Lai, A. (2012). The influence and implications of Chinese culture in the decision to undertake cross-border higher education. Journal of Studies in International education, 16(3), 252-270. https://doi.org/10.1177/1028315311418517 
Chao, C. N., Hegarty, N., Angelidis, J., \& Lu, V. F. (2019). Chinese students' motivations for studying in the United States. Journal of International Students, 7(2), 257-269.

Charmaz, K. (2006). Constructing Grounded Theory: A Practical Guide Through Qualitative Analysis. London: Sage Publications.

Chen Y., \& Hew K.F. (2018) Online Knowledge-Sharing Motivators of Top Contributors in 30 Q\&A Sites. In L. Deng, W. Ma, \& C. Fong (Eds.), New Media for Educational Change. Educational Communications and Technology Yearbook. Springer, Singapore. https://doi.org/10.1007/978-981-10-8896-4_4

Cheung, A. C. K., \& Xu, L. (2015). To return or not to return: Examining the return intentions of mainland Chinese students studying at elite universities in the United States. Studies in Higher Education, 40(9), 1605-1624. https://doi.org/10.1080/03075079.2014.899337

Chung, E., \& Yoon, J. (2017). International students' information needs and seeking behaviours throughout the settlement stages. Libri, 67(2), 119-128. https://doi.org/10.1515/libri-2016-0048

CNNIC. (2017). The 40th China statistical report on internet development. Retrieved from https://cnnic.com.cn/IDR/ReportDownloads/201807/P020180711391069195909.pdf

Corbin, J., \& Strauss, A. (2007). Basics of qualitative research: Techniques and procedures for developing grounded theory (3rd ed.). Thousand Oaks, CA: Sage. https://doi.org/10.4135/9781452230153

Cretacci, M. A., Hendrix, N., Zheng, L., \& Gao, Y. (2018). Is China "Keeping its Powder Dry"? Developing a Cultural Transmission Model of Gun Behaviors and Attitudes. International Journal of Criminal Justice Sciences, 13(1).

Fang, C., \& Zhang, J. (2019). Users' continued participation behavior in social Q\&A communities: A motivation perspective. Computers in Human Behavior, 92, 87-109. https://doi.org/10.1016/j.chb.2018.10.036

Fichman, P. (2011). A comparative assessment of answer quality on four question answering sites. Journal of Information Science, 37(5), 476-486. https://doi.org/10.1177/0165551511415584

Gazan, R. (2015). First-mover advantage in a social Q\&A community. In 2015 48th Hawaii International Conference on System Sciences (pp. 1616-1623). IEEE. https://doi.org/10.1109/HICSS.2015.195

Glass, C., \& Westmont, C. (2013). Comparative effects of belongingness on the academic success and cross-cultural interactions of domestic and international students. International Journal of Intercultural Relations, 38, 106-119. https://doi.org/10.1016/j.jintrel.2013.04.004

Guan, T., Wang, L., Jin, J., \& Song, X. (2018). Knowledge contribution behavior in online Q\&A communities: An empirical investigation. Computers in Human Behavior, 81, 137-147. https://doi.org/10.1016/j.chb.2017.12.023

Hail, H. C. (2015). Patriotism abroad: Overseas Chinese students' encounters with criticisms of China. Journal of Studies in International Education, 19(4), 311-326. https://doi.org/10.1177/1028315314567175

Heng, T. T. (2017). Voices of Chinese international students in USA colleges: 'I want to tell them that...'. Studies in Higher Education, 42(5), 833-850. https://doi.org/10.1080/03075079.2017.1293873

Henze, J., \& Zhu, J. (2012). Current research on Chinese students studying abroad. Research in Comparative and International Education, 7(1), 90-104. https://doi.org/10.2304/rcie.2012.7.1.90

Institute of International Education. (2018). Report of Open Door. Retrieved from https://www.iie.org/Research-and-Insights/Open-Doors

Kim, K., Sin, S., \& Yoo-Lee, E. (2014). Undergraduates' Use of Social Media as Information Sources. College \& Research Libraries, 75(4), 442-457. https://doi.org/10.5860/crl.75.4.442

Krippendorff, K. (2018). Content analysis: An introduction to its methodology. Sage publications.

Lai, L. S., \& To, W. M. (2015). Content analysis of social media: A grounded theory approach. Journal of Electronic Commerce Research, 16(2), 138.

Liao, K. Y. H., \& Wei, M. (2014). Academic stress and positive affect: Asian value and self-worth contingency as moderators among Chinese international students. Cultural Diversity and Ethnic Minority Psychology, 20(1), 107. https://doi.org/10.1037/a0034071

Liu, J., \& Wang, Y. (2016). Information worth spreading: An exploration of information sharing from social Q\&A to other social media platforms. Proceedings of the Association for Information Science and 
Technology, 53(1), 1-5. https://doi.org/10.1002/pra2.2016.14505301112

Lopez, I. Y., \& Bui, N. H. (2014). Acculturation and Linguistic Factors on International Students' Self-Esteem and Language Confidence. Journal of International Students, 4(4), 314-329.

Mamykina, L., Manoim, B., Mittal, M., Hripcsak, G., \& Hartmann, B. (2011). Design lessons from the fastest Q\&A site in the west. In Proceedings of the SIGCHI Conference on Human Factors in Computing Systems (pp. 2857-2866). https://doi.org/10.1145/1978942.1979366

NAFSA: Association of International Educators. (2019). NAFSA international student economic value tool. Washington, DC: NAFSA.

Pata, K., Santos, P., \& Burchert, J. (2016). Social recognition provision patterns in professional Q\&A forums in healthcare and construction. Computers in Human Behavior, 55, 571-583. https://doi.org/10.1016/j.chb.2015.06.046

Rawlings, M., \& Sue, E. (2013). Preparedness of Chinese Students for American Culture and Communicating in English. Journal of International Students, 3(1), 29-40.

Ren, Y., Kraut, R., \& Kiesler, S. (2007). Applying common identity and bond theory to design of online communities. Organization studies, 28(3), 377-408. https://doi.org/10.1177/0170840607076007

Ruble, R. A., \& Zhang, Y. B. (2013). Stereotypes of Chinese international students held by Americans. International Journal of Intercultural Relations, $37(2), \quad 202-211$. https://doi.org/10.1016/j.ijintrel.2012.12.004

Sin, S.-C. J. (2015). Demographic differences International students' information source uses and everyday information seeking challenges. The Journal of Academic Librarianship, 41(4), 466-474. https://doi.org/10.1016/j.acalib.2015.04.003

Teo, H. J., Johri, A., \& Lohani, V. (2017). Analytics and Patterns of Knowledge Creation: Experts at Work in an Online Engineering Community. Computers \& Education, 112, 18-36. https://doi.org/10.1016/j.compedu.2017.04.011

U.S. Department of Homeland Security, I.C.E. (2019). SEVIS by the numbers: Biannual report on international student trends. Washington, D.C.

UNESCO. (2016). Global flow of tertiary level students. Retrieved from http://www.uis.unesco.org/Education/

Vasilescu, B., Serebrenik, A., Devanbu, P., \& Filkov, V. (2014). How social Q\&A sites are changing knowledge sharing in open source software communities. In Proceedings of the 17th ACM conference on CSCW. https://doi.org/10.1145/2531602.2531659

Xu, Q., \& Mocarski, R. (2014). A Cross-Cultural Comparison of Domestic American and International Chinese Students' Social Media Usage. Journal of International Students, 4(4), 374-388.

Yan, K., \& Berliner, D. C. (2011). Chinese international students in the United States: Demographic trends, motivations, acculturation features and adjustment challenges. Asia Pacific Education Review, 12(2), 173-184. https://doi.org/10.1007/s12564-010-9117-x

\section{Copyrights}

Copyright for this article is retained by the author(s), with first publication rights granted to the journal.

This is an open-access article distributed under the terms and conditions of the Creative Commons Attribution license (http://creativecommons.org/licenses/by/4.0/). 